\title{
Một số hợp chất flavonoid phân lập từ lá cây dâu (Morus alba L.) thu hái tại tỉnh Thái Nguyên
}

\author{
Vũ Đức Lợi ${ }^{1, *}$, Đỗ Thị Nghĩa Tình ${ }^{1}$, Bùi Thị Xuân ${ }^{1}$, \\ Vũ Kiều Oanh ${ }^{2}$, Trịnh Nam Trung ${ }^{2}$, Nguyễn Tiến Vững ${ }^{3}$ \\ ${ }^{1}$ Khoa Y Duoọc, Đại học Quốc gia Hà Nội, 144 Xuân Thủy, Cầu Giấy, Hà Nội, Việt Nam \\ ${ }^{2}$ Học viện Quân y, sổ 160 Phùng Hung, Hà Đông, Hà Nội, Việt Nam \\ ${ }^{3}$ Viện Pháp y Quốc gia, số 41 Nguyễn Đình Chiểu, Hai Bà Trung, Hà Nội, Việt Nam
}

Nhận ngày 3 tháng 4 năm 2017

Chỉnh sửa ngày 21 tháng 4 năm 2017; Chấp nhận đăng ngày 14 tháng 6 năm 2017

\begin{abstract}
Tóm tắt: Từ lá của cây dâu (Morus alba $\mathrm{L}$.) thu hái ở tỉnh Thái Nguyên, trên cơ sở sử dụng các phương pháp sắc kí đã phân lập được hai hợp chất flavonoid. Cấu trúc hóa học của hai hợp chất này được xác định là Kaempferol $3-O-\beta$-D-glucopyranosid (1) và Quercetin $-O-\alpha-\mathrm{L}-$ rhamnopyranosid (2) dựa trên các dữ liệu phổ khối lượng và cộng hưởng từ hạt nhân kết hợp so sánh với dữ liệu phổ được công bố trong tài liệu tham khảo. Đây là 2 hợp chất lần đầu tiên được phân lập từ lá cây dâu thu hái tại Việt Nam.
\end{abstract}

Tù khóa: Lá dâu, Morus alba, Kaempferol 3-O- $\beta$-D-glucopyranosid, Quercetin -O- $\alpha$-L-rhamnopyranosid.

\section{1. Đặt vấn đề}

Cây dâu tằm (Morus alba L.) trong sách cổ của Trung Quốc được coi là loài cây quý, bởi nó có rất nhiều công dụng quý đối với con người, vừa có thể làm thuốc trị bệnh, vừa có thể làm thực phẩm bồi bổ cơ thể. Trong đó, lá dâu tằm không chỉ được dùng để chữa các bệnh như tiểu đường, huyết áp cao, rối loạn lipid máu, viêm đường hô hấp, nhức đầu, mờ mắt.....mà còn được dùng với công dụng làm đẹp da, trắng da $[1,2]$. Ngày nay, cùng với sự phát triển của xã hội, nhu cầu làm đẹp của con người tăng lên, đồng thời con người ngày càng có $\mathrm{xu}$ hướng tìm về với tự nhiên để tìm kiếm giải pháp làm đẹp an toàn, hiệu quả. Lá dâu được coi là một trong những nguồn nguyên liệu tự nhiên quý trong

\footnotetext{
* Tác giả liên hệ. ĐT.: 84-989313325.

Email: ducloi82@gmail.com

https://doi.org/10.25073/2588-1132/vnumps.4050
}

việc làm đẹp da, loại bỏ vết thâm nám, tàn nhang trên da. Cho đến nay, các công trình nghiên cứu đã công bố về thành phần hóa học cũng như tác dụng sinh học của lá cây dâu ở Việt Nam còn rất ít. Để góp phần cung cấp những cơ sở tiền đề cho việc ứng dụng nguyên liệu lá dâu trong chăm sóc sức khỏe, bài báo công bố một số thành phần hóa học được nghiên cứu phân lập được từ lá dâu.

\section{2. Đối tượng và phương pháp nghiên cứu}

\section{1. Đối tương nghiên cúu}

Mẫu cây dâu tằm được thu hái vào tháng 6 năm 2016 tại huyện Phổ Yên, tỉnh Thái Nguyên. Mẫu thực vật đã được Viện Sinh thái và Tài nguyên sinh vật giám định tên khoa học là: Morus alba L., họ dâu tằm Moraceae, mẫu được lưu giữ tại Khoa Y Dược, ĐHQGHN. 


\subsection{Dung môi, hóa chất}

Các dung môi dùng trong chiết xuất, phân lập như methanol $(\mathrm{MeOH})$, n-hexan, ethyl acetat $(\mathrm{EtOAc})$, và dicloromethan $(\mathrm{DCM}) \ldots$ dều đạt tiêu chuẩn công nghiệp và được chưng cất lại trước khi dùng. Dung môi phân tích gồm $\mathrm{MeOH}$, n-hexan, EtOAc, $\mathrm{H}_{2} \mathrm{O}$ dùng để phân tích sắc ký đều đạt tiêu chuẩn phân tích. Pha tĩnh dùng trong sắc ký cột là silicagel pha thường $(0,040-0,063 \mathrm{~mm}$, Nicalai Tesque Inc., Nhật Bản), YMC ODS-A $(50 \mu \mathrm{m}$, YMC Co. Ltd., Nhật Bản). Bản mỏng tráng sẵn trên đế nhôm loại pha thường Kieselgel $60 \mathrm{~F}_{254}$ và pha đảo TLC Silica gel 60 RP-18 $F_{254 \mathrm{~S}}$ (Merck, Damstadt, Đức). Phát hiện chất bằng đèn tử ngoại ở hai bước sóng $254 \mathrm{~nm}$ và $365 \mathrm{~nm}$ hoặc dùng thuốc thử là dung dịch $\mathrm{H}_{2} \mathrm{SO}_{4} 10 \%$ hơ nóng để phát hiện vết chất.

\subsection{Thiết bị, dụng cu}

- Sắc ký cột: sắc ký cột sử dụng silicagel cõ hạt $0,063-0,200 \mathrm{~mm}$ (Merck) và cỡ hạt 0,040 $0,063 \mathrm{~mm}$ (Merck) với các loại cột sắc ký có kích cỡ khác nhau.

- Phổ cộng hưởng tù hạt nhân: NMR được ghi trên máy Bruker Avance $500 \mathrm{MHz}$ tại Viện Hóa học, Viện Hàn lâm Khoa học và Công nghệ Việt Nam.

- Phổ khối ESI-MS: đo trên máy AGILENT 1260 Series LC-MS ion Trap (Agilent Technologies, Hoa Kỳ)

- Nhiệt độ nóng chảy: đo trên máy SMP10 BioCote, Khoa Y Dược, ĐHQGHN.

- Góc quay cực riêng: đo trên máy PLR-4, MRC scientific instruments, Khoa Y Dược, ĐHQGHN.

\subsection{Chiết tách và phân lập các hợp chất}

Lá dâu được thu hái, rửa sạch, phơi và sấy khô ở $50^{\circ} \mathrm{C}$, nghiền nhỏ thu được bột thô. Lấy $6,0 \mathrm{~kg}$ bột khô (đã trừ độ ẩm) đem ngâm chiết với 9,0 lít methanol/lần $\mathrm{x} 4$ lần ở nhiệt độ phòng, mỗi lần 48 giờ. Các dịch chiết được gom lại, lọc qua giấy lọc và cất loại dung môi dưới áp suất giảm thu được $520 \mathrm{~g}$ cắn chiết methanol. Cắn chiết được phân bố vào nước cất vừa đủ và tiến hành chiết lần lượt với $\mathrm{n}$ hexan, ethylacetat. Các dịch chiết n-hexan, ethylacetat và phần nước còn lại được cất thu hồi dung môi dưới áp suất giảm thu được các cắn $\mathrm{n}$-hexan $(\mathrm{A}, 60 \mathrm{~g})$, cắn ethylacetat $(\mathrm{B}, 75$ $\mathrm{g})$ và cắn nước $(\mathrm{C}, 42 \mathrm{~g})$.

Cắn ethylacetat $(\mathrm{B}, 50 \mathrm{~g})$ được hòa tan trong lượng dung môi vừa đủ và trộn với silicagel $(150 \mathrm{~g})$, sau đó cất loại dung môi để được dạng bột tơi, tiến hành sắc ký cột với chất hấp phụ silica gel, kích thước cột $60 \mathrm{~cm}$ x $10 \mathrm{~cm}$ (chiều dài $\mathrm{x}$ đường kính cột), dung môi rửa giải dicloromethan: methanol với độ phân cực của dung môi tăng dần (từ 20:1 đến $0: 1$ ) thu được 4 phân đoạn $\mathbf{B} 1(6,0 \mathrm{~g}), \mathbf{B} 2(7,5 \mathrm{~g}), \mathbf{B 3}(12,0 \mathrm{~g})$ và B4 $(10,0 \mathrm{~g})$. Phân tách phân đoạn $\mathbf{B 3}$ trên sắc ký cột với chất hấp phụ pha đảo (ODS) sử dụng YMC-gel, kích thước cột $80 \mathrm{~cm} \times 3 \mathrm{~cm}$ (chiều dài chất nhồi $70 \mathrm{~cm}$ ) và kích thước cột $80 \mathrm{~cm} \times 1,5 \mathrm{~cm}$ (chiều dài chất nhồi $70 \mathrm{~cm}$ ), sử dụng hệ dung môi rửa giải aceton: nước $(2: 5$, v:v) thu được 4 phân đoạn nhỏ là $B 3.1$ (2,1 g), B3.2 (3,1 g), B3.3 (1,9 g), B3.4 (3,6 g).

Phân đoạn $\mathrm{B} 3.1$ được tinh chế bằng $\mathrm{CC}$ pha đảo với hệ dung môi rửa giải $\mathrm{MeOH}-\mathrm{H}_{2} \mathrm{O}(2: 1)$ thu được chất rắn màu vàng ký hiệu là hợp chất 2 (41mg). Phân đoạn B3.2 được phân tách bằng sắc ký cột silicagel pha thường rửa giải bằng hệ dung môi chloroform/methanol $(5 / 2, \mathrm{v} / \mathrm{v})$ thu được hợp chất 1 (48 mg).

\section{Kết quả và bàn luận}

\section{Hợp chất 1: Kaempferol 3-O- $\beta-D-$ glucopyranosid}

Chất tinh thể hình kim, màu vàng. Nhiệt độ nóng chảy $178-179^{\circ} \mathrm{C}$.

Độ quay cực: $[\alpha]_{\mathrm{D}}^{25}=+16,9 \quad(c=0,65$, $\mathrm{MeOH})$. ESI-MS: $m / z \quad 447[\mathrm{M}-\mathrm{H}]^{-}$và 471 $[\mathrm{M}+\mathrm{Na}]^{+} \mathrm{CTPT}: \mathrm{C}_{21} \mathrm{H}_{20} \mathrm{O}_{11}$; KLPT M =448; Số liệu phổ ${ }^{1} \mathrm{H}-\mathrm{NMR}$ và ${ }^{13} \mathrm{C}$-NMR (DMSO- $d_{6}$ ) được trình bày ở Bảng 1 . 
Bảng 1. Dữ liệu phổ NMR của hợp chất 1 và chất so sánh $\mathrm{M}$

\begin{tabular}{lllllll}
\hline Vi trí & DEPT & $\begin{array}{l}\delta \mathrm{C}(\mathrm{M}) \\
\mathrm{C}\end{array}$ & $\begin{array}{l}\delta_{\mathrm{C}}(1) \mathrm{ppm} \\
\mathrm{ppm}\end{array}$ & $\begin{array}{l}\delta \mathrm{H}(1) \mathrm{ppm}, \\
J: \mathrm{Hz}\end{array}$ & $\begin{array}{l}\delta \mathrm{H}(\mathrm{M}) \mathrm{ppm}, \\
J: \mathrm{Hz}\end{array}$ & $\mathrm{HMBC}(1)(\mathrm{H} \rightarrow \mathrm{C})$ \\
\hline 2 & $\mathrm{C}$ & 156,7 & 156,7 & - & - & \\
3 & $\mathrm{C}$ & 133,5 & 133,4 & - & - & \\
4 & $\mathrm{C}$ & 177,6 & 177,7 & - & - & \\
5 & $\mathrm{C}$ & 160,8 & 161,4 & - & $6,22(\mathrm{~d}, 2,0)$ & $7,5,10,8$ \\
6 & $\mathrm{CH}$ & 99,3 & 98,9 & $6,24(\mathrm{~d}, 2,0)$ & - & \\
7 & $\mathrm{C}$ & 162,2 & 164,4 & - & - & \\
8 & $\mathrm{CH}$ & 94,5 & 94,0 & $6,43(\mathrm{~d}, 2,0)$ & $6,41(\mathrm{~d}, 2,0)$ & $7,9,10,6$ \\
9 & $\mathrm{C}$ & 156,0 & 156,6 & - & - & \\
10 & $\mathrm{C}$ & 105,5 & 104,3 & - & - & \\
$1^{\prime}$ & $\mathrm{C}$ & 120,7 & 121,2 & - & - & \\
$2^{\prime}, 6^{\prime}$ & $\mathrm{CH}$ & 130,9 & 131,2 & $8,05(\mathrm{dd}, 1,5,7,0)$ & $8,06(\mathrm{dd}, 1,5,7,0)$ & $4^{\prime}, 2^{\prime}, 2^{\prime}, 6^{\prime}$ \\
$3^{\prime}, 5^{\prime}$ & $\mathrm{CH}$ & 115,1 & 115,4 & $6,87(\mathrm{dd}, 1,5,7,0)$ & $6,85(\mathrm{dd}, 1,5,7,0)$ & $4^{\prime}, 1^{\prime}, 3^{\prime}, 5^{\prime}$ \\
$4^{\prime}$ & $\mathrm{C}$ & 160,2 & 160,1 & - & - & \\
$1^{\prime \prime}$ & $\mathrm{CH}$ & 100,8 & 101,1 & $5,44(\mathrm{~d}, 7,5)$ & $5,46(\mathrm{~d}, 7,5)$ & 3 \\
$2^{\prime \prime}$ & $\mathrm{CH}$ & 74,2 & 74,4 & $3,18(\mathrm{~m})$ & $3,16(\mathrm{~m})$ & $1^{\prime \prime}, 3^{\prime \prime}$ \\
$3^{\prime \prime}$ & $\mathrm{CH}$ & 77,5 & 77,6 & $3,08(\mathrm{~m})$ & $3,09(\mathrm{~m})$ & $4^{\prime \prime}$ \\
$4^{\prime \prime}$ & $\mathrm{CH}$ & 69,9 & 70,1 & $3,09(\mathrm{~m})$ & $3,06(\mathrm{~m})$ & $5^{\prime \prime}, 3^{\prime \prime}$ \\
$5^{\prime \prime}$ & $\mathrm{CH}$ & 76,4 & 76,6 & $3,25(\mathrm{~m})$ & $3,26(\mathrm{~m})$ & \\
$6^{\prime \prime}$ & $\mathrm{CH} 2$ & 60,8 & 61,1 & $3,33(\mathrm{~m})$ & $3,35(\mathrm{~m})$ & \\
& & & & $3,56(\mathrm{dd}, 5,5,11,0)$ & $3,53(\mathrm{dd}, 5,5,11,0)$ & \\
\hline
\end{tabular}

$\delta \mathrm{C}(\mathrm{M}), \delta \mathrm{H}(\mathrm{M})$ của kaempferol 3-O- $\beta$-D-glucopyranoside $[3,4]$.<smiles>O=c1c(OC2OC(CO)C(O)C(O)C2O)c(-c2ccc(O)cc2)oc2cc(O)cc(O)c12</smiles>

Hình 1. Cấu trúc hóa học của hợp chất 1.

Hợp chất 1 thu được dưới dạng tinh thể hình kim, màu vàng. Phổ khối lượng ESI-MS của 1 xuất hiện tín hiệu tại $m / z 447[\mathrm{M}-\mathrm{H}]^{-}$và $471[\mathrm{M}+\mathrm{Na}]^{+}$ứng với khối lượng phân tử là 448, công thức phân tử của hợp chất này có thể là $\mathrm{C}_{21} \mathrm{H}_{20} \mathrm{O}_{11}$. Trên phổ ${ }^{1} \mathrm{H}-\mathrm{NMR}$ của 1 xuất hiện: Tín hiệu proton anome tại $\delta_{\mathrm{H}} 5,44(1 \mathrm{H}$, $\mathrm{d}, J=7,5 \mathrm{~Hz})$ gợi ý trong 1 có mặt 1 phần đường; cặp tín hiệu doublet tại $6,24(\mathrm{~d}, J=2,0$ $\mathrm{Hz})$ và $6,43(\mathrm{~d}, J=2,0 \mathrm{~Hz})$ điển hình cho hai proton ở vị trí $\mathrm{C}-6$ và $\mathrm{C}-8$ của vòng $\mathrm{A}$ của hợp chất flavonol; cặp hai tín hiệu doublet khác tại $\delta_{\mathrm{H}} 6,87(\mathrm{dd}, J=1,5,7,0 \mathrm{~Hz})$ và $8,05(\mathrm{dd}, J$ $=1,5,7,0 \mathrm{~Hz}$ ) đặc trưng cho vòng thơm $\mathrm{B}$ thế para. Phổ ${ }^{13} \mathrm{C}$-NMR của 1 xuất hiện tín hiệu của 21 nguyên tử cacbon, trong đó có 6 tín hiệu tại $\delta_{\mathrm{C}} 101,1 \quad\left(\mathrm{C}-1^{\prime \prime}\right), 74,4\left(\mathrm{C}-2^{\prime \prime}\right), 77,6$ (C-3"), 70,1 (C-4"), 76,6 (C-5"), 61,1 (C-6") khẳng định sự có mặt của phần đường glucose và tín hiệu của 15 nguyên tử cacbon thuộc vào khung flavonol có vòng $\mathrm{B}$ thế para. So sánh các dữ liệu phổ NMR của 1 với hợp chất kaempferol 3-O- $\beta$ - D-glucopyranoside thấy sự 
trùng hợp $[3,4]$. Tương tác $\mathrm{HMBC}$ giữa $\mathrm{H}-1 "$ $\left(\delta_{\mathrm{C}} 5,44\right)$ và $\mathrm{C}-3\left(\delta_{\mathrm{C}} 133,4\right)$ gợi ý phần $O-\beta$-Dglucopyranosyl tại C-3 của flavonol. Như vậy, có thể khẳng định hợp chất 1 là kaempferol 3-O- $\boldsymbol{\beta}$-D-glucopyranoside. Hợp chất này có mặt trong nhiều loài thực vật và có hoạt tính chống ôxi hóa mạnh.
Hợp chất 2: Quercetin 3-O- $\alpha-L-$ rhamnopyranosid

Tinh thể màu vàng nhạt, $\mathrm{M}=448, \mathrm{M}_{\mathrm{p}}=182$ $183^{\circ} \mathrm{C} ; R_{f}=0,45\left(\mathrm{CHCl}_{3}-\mathrm{MeOH}, 85: 15\right) ; E S I-$ $M S \mathrm{~m} / z$ : 447,0 [M-H] $]^{-}$. Phổ ${ }^{1} H-N M R$ (500MHz), ${ }^{13} \mathrm{C}-N M R$ (125MHz) đo trong $\mathrm{CD}_{3} \mathrm{OD}$ và phổ DEPT của chất 2 được trình bày ở bảng 2 :

Bảng 2. Dữ liệu phổ NMR của hợp chất 2 và chất so sánh $\mathrm{K}$

\begin{tabular}{cccccc}
\hline Vị trí C & DEPT & $\begin{array}{c}\delta_{\mathrm{H}}(\mathrm{K}) \mathrm{ppm}, \\
J: \mathrm{Hz}\end{array}$ & $\begin{array}{c}\delta_{\mathrm{H}}(2) \mathrm{ppm}, \\
J: \mathrm{Hz}\end{array}$ & $\begin{array}{c}\delta_{\mathrm{C}}(\mathrm{K}) \\
\mathrm{ppm}\end{array}$ & $\begin{array}{c}\delta_{\mathrm{C}}(2) \\
\mathrm{ppm}\end{array}$ \\
\hline 2 & $\mathrm{C}$ & - & - & 158,1 & 158,5 \\
3 & $\mathrm{C}$ & - & - & 136,0 & 136,2 \\
4 & $\mathrm{C}$ & - & - & 179,3 & 179,7 \\
5 & $\mathrm{C}$ & - & - & 159,1 & 159,3 \\
6 & $\mathrm{CH}$ & $6,10 d(1,8)$ & $6,22 d(2,0)$ & 99,5 & 99,9 \\
7 & $\mathrm{C}$ & - & - & 165,8 & 165,9 \\
8 & $\mathrm{CH}$ & $6,29 d(1,8)$ & $6,39 d(2,0)$ & 94,3 & 94,7 \\
9 & $\mathrm{C}$ & - & - & 163,0 & 158,5 \\
10 & $\mathrm{C}$ & - & - & 105,5 & 105,9 \\
$1^{\prime}$ & $\mathrm{C}$ & - & $7,36 d(2,5)$ & 122,5 & 123,0 \\
$2^{\prime}$ & $\mathrm{CH}$ & $7,57 d(1,9)$ & - & 116,2 & 116,4 \\
$3^{\prime}$ & $\mathrm{C}$ & - & - & 146,2 & 146,4 \\
$4^{\prime}$ & $\mathrm{C}$ & - & $6,93 d(8,0)$ & 119,5 & 149,8 \\
$5^{\prime}$ & $\mathrm{CH}$ & $6,77 d(8,2)$ & $7,33 d d(2,0 ; 8,5)$ & 122,7 & 117,0 \\
$6^{\prime}$ & $\mathrm{CH}$ & $7,53 d d(1,9 ; 8,2)$ & $5,37 d(1,5)$ & 103,4 & 103,9 \\
$1^{\prime \prime}$ & $\mathrm{CH}$ & $5,01 d(2,0)$ & $4,25 d d(1,7 ; 3,3)$ & 71,8 & 72,0 \\
$2^{\prime \prime}$ & $\mathrm{CH}$ & & $3,77 d d(3,5 ; 9,5)$ & 72,0 & 72,1 \\
$3^{\prime \prime}$ & $\mathrm{CH}$ & $4,22-3,14$ & $3,36 d d(2,3,9,58)$ & 73,1 & 73,3 \\
$4^{\prime \prime}$ & $\mathrm{CH}$ & & $3,44 d d(6,0 ; 9,5)$ & 71,7 & 71,9 \\
$5^{\prime \prime}$ & $\mathrm{CH}$ & & $0,96 d(6,5)$ & 17,5 & 17,6 \\
$6^{\prime \prime}$ & $\mathrm{CH}$ & $1,02 d(6,0)$ & & & \\
\hline
\end{tabular}

Ghi chú: $\delta_{\mathrm{H}}(\mathrm{K})$ đo trong $\mathrm{CD}_{3} \mathrm{OD}$ ở $400 \mathrm{MHz}, \delta_{\mathrm{C}}(\mathrm{K})$ đo trong $\mathrm{CD}_{3} \mathrm{OD}$ ở $100 \mathrm{MHz}$ của chất quercitrin $[5,6]$.

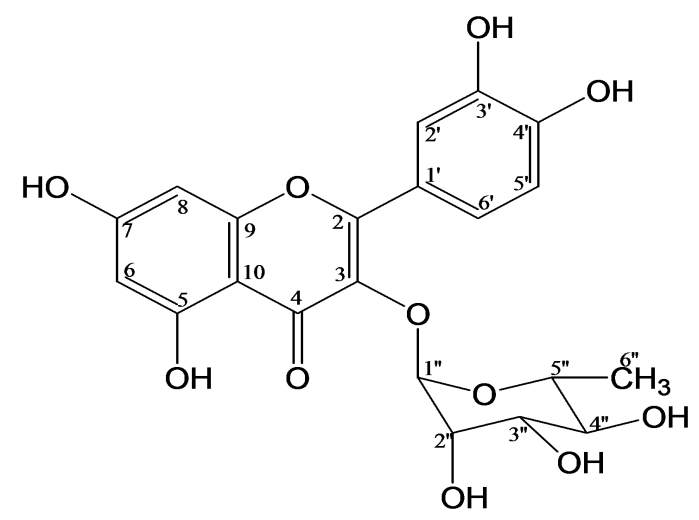

Hình 2. Cấu trúc của hợp chất 2. 
Hợp chất 2 nhận được dưới dạng chất bột có màu vàng đặc trưng cho nhóm chất flavonoid. Phổ ESI-MS của hợp chất 2 xuất hiện pic ion tại $m / z: 447[\mathrm{M}-\mathrm{H}]$ ], tương ứng với khối lượng phân tử $\mathrm{M}=448$, phù hợp với công thức phân tử $\mathrm{C}_{21} \mathrm{H}_{20} \mathrm{O}_{11}$.

Phổ ${ }^{1} \mathrm{H}-\mathrm{NMR}$ của hợp chất 2 xuất hiện hai vùng phổ đặc trưng, một vùng ở trường khá thấp với hai tín hiệu của hai proton nằm ở vị trí meta với nhau thuộc vòng $A$ tại tại 6,22 và 6,39 $(\mathrm{d}, J=2,0 \mathrm{~Hz})$ và ba tín hiệu cộng hưởng với tương tác spin coupling dạng $\mathrm{ABX}$ của vòng $\mathrm{B}$ thế ở các vị trí 1,3,4 tại 7,36 $(1 \mathrm{H}, \mathrm{d}, J=2,5, \mathrm{H}-$ $\left.2^{\prime}\right), 6,93(1 \mathrm{H}, \mathrm{d}, J=8,0, \mathrm{H}-5 ') ; 7,33(1 \mathrm{H}, \mathrm{dd}, J=$ 2,$\left.0 ; 8,5 ; \mathrm{H}-6^{\prime}\right)$; Vùng trường cao hơn là các tín hiệu của một phân tử đường. Tín hiệu của proton anome tại $5,37(1 \mathrm{H}, \mathrm{d}, J=1,5 ; \mathrm{H}-1$ "), tín hiệu điển hình của nhóm methyl bậc một dưới dạng doublet tại $0,96(3 \mathrm{H}, \mathrm{d}, J=6,5 \mathrm{~Hz}, \mathrm{H}-6 ")$ và bốn proton của các nhóm oxymethin tại 3,77 $(1 \mathrm{H}, \mathrm{dd}, J=3,5 ; 9,5 ; \mathrm{H}-3 ") ; 4,25(1 \mathrm{H}, \mathrm{dd}, J=$ $1,7 ; 3,3 ; \mathrm{H}-2 ") ; 3,36(1 \mathrm{H}, \mathrm{dd}, \mathrm{J}=2,3,9,58 ; \mathrm{H}-$ 4"); 3,44(1H, dd, $\mathrm{J}=6,0 ; 9,5 ; \mathrm{H}-5 ")$ cho thấy cấu trúc của 2 có một phân tử đường rhamnose.

Phổ ${ }^{13} \mathrm{C}-\mathrm{NMR}$ của 2 xuất hiện tín hiệu của 21 nguyên tử carbon, trong đó có 15 tín hiệu của khung flavon và 6 tín hiệu của một phân tử đường rhamnose. Các tín hiệu của vòng $\mathrm{B}$ lần lượt tại 123,0 (C, C-1'); 116,4 (CH, C-2'); 146,4 (C, C-3'); 149,8(C, C-4'); 117,0 (CH, C-5'); 122,9 $(\mathrm{CH}, \mathrm{C}-6$ '). Nhóm carbonyl xuất hiện tại 179,7 (C-4), hai tín hiệu $\mathrm{CH}$ điển hình tương ứng với các vị trí $\mathrm{C}-6$ và $\mathrm{C}-8$ của vòng $\mathrm{A}$ tại $99,9(\mathrm{C}-6)$ và 94,7 (C-8); tín hiệu của carbon anome tại 103,5 (C-1"), nhóm methyl tại 17,6 (C-6") và bốn tín hiệu $\mathrm{CH}$ nối với oxi của phân tử đường rhamnose tại 72,0 (C-2"); 72,1 (C-3"); 73,3 (C-4"); 71,9 (C5"). Từ các kêt quả nêu trên, đối chiếu với dữ liệu đã công bố $[5,6]$ hợp chất 2 được xác định là quercetin 3-O- $\alpha$-L-rhamnopyranosid.

\section{Kết luận}

Bằng các phương pháp sắc ký kết hợp với các phương pháp phân tích phổ hiện đại (MS, NMR), từ phân đoạn dịch chiết ethylacetat của lá cây dâu đã phân lập, xác định cấu trúc phân tử 2 hợp chất nhóm flavonoid là kaempferol 3$O-\beta$-D-glucopyranoside (1) và quercetin $3-O-\alpha-$ L-rhamnopyranosid (2). Các kết quả trên cũng mở ra những hướng nghiên cứu sâu hơn nhằm hướng tới mục tiêu tìm ra hoạt chất chính có khả năng ứng dụng làm chất chuẩn trong kiểm nghiệm. Đồng thời cần tiếp tục thực hiện các nghiên cứu bổ sung về hàm lượng và tác dụng sinh học của các hợp chất phân lập được, nhằm minh chứng cho công dụng và góp phần định hướng sử dụng cây dâu hiệu quả hơn.

\section{Lời cảm ơn}

Nghiên cứu này được tài trợ bởi Quỹ Khoa học Công nghệ Đại học Quốc Gia Hà Nội, đề tài "Phát triển sản phẩm thực phẩm chức năng và mỹ phẩm làm sáng da, chống nám từ nguyên liệu thiên nhiên Việt Nam", mã số: QG.16.86

\section{Tài liệu tham khảo}

[1] Đỗ Tất Lợi (2001). Những cây thuốc và vị thuốc Việt Nam. NXB Y học, tr. 628-629.

[2] Viện Dược liệu (2004). Cây thuốc và động vật làm thuốc ở Việt Nam. NXB Khoa học và Kỹ thuật, tr. 462-468.

[3] Ayse Kuruzum-uz, Zühal Guvenalp, Cavit Kazaz (2013), Phenolic compounds from the roots of Anchusa azurea var. azurea, Turk J Pharm Sci, 10 (2), 177-184.

[4] Choi J, Kang HJ, Kim SZ, Kwon TO, Jeong SI, Jang SI. (2013), Antioxidant effect of astragalin isolated from the leaves of Morus alba L. against free radical-induced oxidative hemolysis of human red blood cells. Arch Pharm Res, 36(7), pp. 912-7.

[5] Lee E. H., Song D.-G., Lee J. Y., et al. (2009), "Flavonoids from the leaves of Thuja orientalis inhibit the aldose reductase and the formation of advanced glycation endproducts", Journal of the Korean Society for Applied Biological Chemistry, 52(5), pp. 448-455.

[6] Wang K.J., Yang C.R., and Zhang Y.J. (2007), "Phenolic antioxidants from Chinese toon (fresh young leaves and shoots of Toona sinensis)", Food Chemistry, 101(1), pp. 365-371. 


\title{
Flavonoids Isolated from the Leaf of Morus alba L. Collected in Thai Nguyen Province
}

\author{
Vu Duc Loi ${ }^{1}$, Do Thi Nghia Tinh ${ }^{1}$, Bui Thi Xuan ${ }^{1}$, \\ $\mathrm{Vu}$ Kieu Oanh${ }^{2}$, Trinh Nam Trung ${ }^{2}$, Nguyen Tien Vung ${ }^{3}$ \\ ${ }^{1}$ VNU School of Medicine and Pharmacy, 144 Xuan Thuy, Cau Giay, Hanoi, Vietnam \\ ${ }^{2}$ Vietnam Militery Medical University, 160 Phung Hung, Ha Dong District, Hanoi, Vietnam \\ ${ }^{3}$ National Institute of Forensic Medicine, 41 Nguyen Dinh Chieu, Hai Ba Trung District, Hanoi, Vietnam

\begin{abstract}
From the leaf of Morus alba L. collected in Thai Nguyen province, using chromatography methods resulted in the isolation of two flavonoids. Their structures were identified as kaempferol 3-O- $\beta$-D-glucopyranoside (1), quercetin 3-O- $\alpha$-L-rhamnopyranoside (2) on the basis of spectroscopic data including mass spectrometry and nuclear magnetic resonance spectra together with comparison with those reported in the literature. This is the first report of flavonoid components from the leaf of Morus alba L. colected in Vietnam.
\end{abstract}

Keywords: Morus alba, kaempferol 3-O- $\beta$-D-glucopyranoside, quercetin 3-O- $\alpha$-L-rhamnopyranoside. 